\title{
Selecting Objects for ALVOT
}

\author{
Miguel Angel Medina-Pérez ${ }^{1}$, Milton García-Borroto², \\ Yenny Villuendas-Rey ${ }^{1}$, and José Ruiz-Shulcloper ${ }^{3}$ \\ ${ }^{1}$ University of Ciego de Ávila, Cuba \\ (migue, yennyv) abioplantas.cu \\ http: / /www.unica.cu \\ ${ }^{2}$ Bioplants Center, UNICA, C. de Ávila, Cuba \\ mil@ioplantas.cu \\ http: / / www.bioplantas.cu \\ ${ }^{3}$ Advanced Technologies Applications Center, MINBAS, Cuba \\ jshulcloper@cenatav.co.cu \\ http: //www. cenatav.co.cu/
}

\begin{abstract}
ALVOT is a model of supervised classification based on partial precedences. In this paper a new object selection method based on a voting procedure for ALVOT is proposed. The method was developed for dealing with databases having objects described by features that are not exclusively numeric or categorical. A comparative numerical experiment was performed with different algorithms of object selection. The experimental results show a good performance of the proposed method with respect to the other algorithms.
\end{abstract}

\section{Introduction}

To achieve high-quality results, supervised classifiers need a good training matrix. It is usually accomplished by removing "noisy" as well as redundant objects. Several techniques have been developed to deal with these two problems, increasing the quality of classification: editing and condensing methods respectively [1].

On the other hand, in real world problems, sometimes the objects are described simultaneously in terms of qualitative and quantitative features. Some of the object descriptions could also be incomplete (missing values). In these cases, the tools of the Logical Combinatorial Pattern Recognition (LCPR) [2] should be used.

ALVOT [3, 4], introduced by Zhuravlev, is a model of supervised classification in the LCPR. The original algorithm has been applied to different real world situations, with the introduction of new similarity functions. Also, the fuzzy set theory concepts have been used in order to adequately model some problems in geosciences, medicine, and others $[5,6]$.

ALVOT is based on partial precedence. Partial precedence is the principle of calculating the similarity between objects using comparisons between their partial descriptions. A partial description is a combination of features with a clear meaning in the problem domain. This is the way that physicians, and other natural scientists, establish comparisons among objects in real world problems [4].

For a new object to be classified, many partial comparisons with all the objects in the training matrix have to be calculated. This can be very time consuming, while the 
cardinality of the matrix increases. That is why we propose an object selection method, which improves the quality of classification with ALVOT.

\section{Previous Works}

The first editing method for ALVOT was introduced in [7, 8]. This method uses a set of genetic algorithms to select the support sets system, the features weights, and the objects in the training matrix. Nevertheless, there are several problems where this optimization procedure can not be applied, because the parameters have a meaning in the problem definition domain. For example, the specialist might determine the support sets system and assign the feature weights according to his background knowledge. He can also use some procedures, with a comprehensive meaning in his model. Based on these facts, we compared our algorithm only with the object selection procedure.

The chromosomes used by the genetic algorithm were binary strings consisting of $m$ bits, one for each object, representing the subsets of those which are selected. The $i^{\text {th }}$ bit has value 1 when the respective element is included, and 0 otherwise.

In general, the algorithm works as follows:

1 The initial population is generated randomly. The population size and iteration number are input parameters of the algorithm.

2 The population's individuals are sorted according to their fitness. The first and last individuals are crossed, the second is crossed with the penultimate and this process is repeated until finishing the population. They are crossed using a 1-point crossover operator in the middle of the individual. The fitness function is the ratio of well classified objects.

3 For each individual in the population the mutation operator is applied. It takes randomly an individual's gene and changes its value. The fitness is evaluated for this new population.

4 The original individuals together with those obtained by crossing and mutation are sorted in descending order according to their fitness and those with highest fitness are chosen (taking into account the population size). The new population is used in the next iteration of the algorithm.

This method has an important random component, so two different executions of the algorithm with the same data could have dramatically different results. Besides, the results reached by this procedure lack of a comprehensive meaning in the problem domain. As pointed out by Kuncheva and Bezdek [9] selecting prototypes by Random Searches and Genetic Algorithms could be computationally demanding and, for large data sets, may be infeasible.

\section{A First Approach to a New Object Selection Method for ALVOT}

For selecting objects for ALVOT we can apply a classical editing methods based on NN rule. An analogue solution were reported by Decaestecker [10] and Konig et. al [11], in which the training matrix is edited for a Radial Based Function network, using a procedure originally designed for k-NN. A deep study about editing methods is outside of the scope of this paper, but a revision can be found in [1]. 
Considering that we are working with MID, in this paper we use the Compact Set Editing method(CSE) [12], an extension of Wilson's [13] Edited Nearest Neighbor rule (ENN) for MID, and a modification of the ENN using ALVOT as classifier (EALVOT).

Despite these procedures can be applied, as we can see below, a better solution could be achieved considering the way ALVOT works.

\section{Voting Based Object Selection (VOS)}

Any algorithm of ALVOT $A$ works in six stages:

Stage 1.- Determine the support sets system $\Omega_{A}$, which is a set of subsets of features.

Stage 2.- Find out the feature comparison criteria and similarity function between sub-descriptions of objects $\beta\left(\Omega O, \Omega O_{i}\right), \Omega \in \Omega_{A} . \Omega O$ is a sub-description of object $O$ using only features in $\Omega$; and $\beta$ is a similarity function.

Stage 3.- Rule for evaluating the partial similarity between a fixed sub-description of a new object and the corresponding sub-descriptions of previously classified objects $\Gamma_{\Omega}\left(O_{p}, O\right)$ (partial evaluation by rows).

Stage 4.- Rule for summarizing the partial evaluation by rows of a fixed subdescription of a new object for each class $\Gamma_{\Omega}^{j}(O)$ (partial evaluation by classes).

Stage 5.- Rule for summarizing all partial evaluations by class with respect to the whole support sets system of a new object $\Gamma^{j}(O)$ (total evaluation by classes).

Stage 6.- Decision-making rule based on the total evaluation by classes $\Gamma_{A}$.

In this paper we introduce a new method to select objects for ALVOT, named Voting based Object Selection (VOS). Objects are selected according to their voting power in the training data, which is calculated with the following expression:

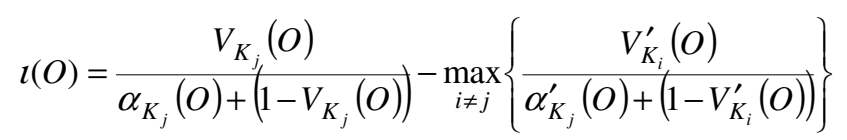

where:

- $V_{K_{j}}(O)=\frac{1}{\left|K_{j}\right|-1} \sum_{\substack{O_{i} \in K_{j} \\ O_{i} \neq O}} I_{K_{j}}\left(O, O_{i}\right) ;$ summarizes the votes that $O$ gives to the objects of its own class $\left(K_{j}\right)$.

- $I_{K_{j}}\left(O, O_{i}\right)=\frac{1}{\left|\Omega_{A}\right|} \sum_{\Omega \in \Omega_{A}} \frac{1}{\left|K_{j}\right|-1} \cdot \rho(O) \cdot \rho(\Omega) \cdot \beta\left(\Omega O, \Omega O_{i}\right) ; \quad$ a voting function obtained from ALVOT stages 3 to 5. This function express the vote that the object 
$O$ gives to $O_{i}$, being $\rho\left(O_{p}\right)$ the weight of the object $O_{p}, \rho(\Omega)=\sum_{\chi_{i} \in \Omega} \rho\left(\chi_{i}\right)$, and $\rho\left(\chi_{i}\right)$ the weight of the feature $\chi_{i}$.

- $\alpha_{K_{j}}(O)=\frac{1}{\left|K_{j}\right|-1} \sum_{\substack{O_{i} \in K_{j} \\ O \neq O_{i}}}\left|V_{K_{j}}(O)-I_{K_{j}}\left(O, O_{i}\right)\right|$ the variance between the mean $V_{K_{j}}(O)$ and the vote that the object $O$ gives to the other objects in $K_{j}$; so when the variance decreases, the values of $l(O)$ increases.

- $V_{K_{i}}^{\prime}(O)=\frac{1}{\left|K_{i}\right|} \sum_{O_{h} \in K_{i}} I_{K_{i}}^{\prime}\left(O, O_{h}\right)$; analogous to $V_{K_{j}}(O)$, but $O \notin K_{i}$.

- $I_{K_{i}}^{\prime}\left(O, O_{h}\right)=\frac{1}{\left|\Omega_{A}\right|} \sum_{\Omega \in \Omega_{A}} \frac{1}{\left|K_{j}\right|} \cdot \rho(O) \cdot \rho(\Omega) \cdot \beta\left(\Omega O, \Omega O_{h}\right)$, analogous to $I_{K_{j}}\left(O, O_{i}\right)$, but $O \notin K_{i}$.

- $\alpha_{K_{i}}^{\prime}(O)=\frac{1}{\left|K_{i}\right|} \sum_{O_{h} \in K_{i}}\left|V_{K_{i}}^{\prime}(O)-I_{K_{i}}^{\prime}\left(O, O_{h}\right)\right|$; analogous to $\alpha_{K_{j}}(O)$, but $O \notin K_{i}$.

Let be $Q_{A}(S, V)=W_{a} \cdot A c c-W_{o} \cdot \frac{|S|}{|T|}$ a quality function; where $W_{a}$ is the weight associated to the accuracy obtained from classifying the objects in $V$ using $S$ as training matrix; $T$ the original training matrix; and $W_{o}$, the weight associated to the reduction ratio.

The algorithm VOS works as follows:

Step 0: Calculate $Q_{A}(T, V)=q_{0}$, the quality of $A$ classifying the validation matrix $V$ using the training matrix $T$.

Step 1: Calculate the voting power of each object using (1).

Step 2: Sort descendent the objects per class according to $t(O)$.

Step 3: Calculate $c_{0}=\min _{i \in 1 \ldots r}\left\{\left\{\left\{O \in K_{i}: \imath(O)>0\right\}\right\}\right.$, where $r$ is the total amount of classes. This value is the minimal number of objects in any class with a positive $\imath(O)$. Select an initial solution $S_{0}$ taking the first $c_{0}$ objects from each class.

Step 4: Calculate $q_{1}$, the quality of $A$ classifying $V$ using $S_{0}$ as the training matrix.

Step 5: If $q_{1} \geq q_{0}$, keep removing from $S_{0}$ the object with less $\imath(O)$ per class, while the quality is above $q_{0}$ and no empty class is obtained.

Step 6: If $q_{1}<q_{0}$, keep adding from $T \backslash S_{0}$ to $S_{0}$ the object with greater $t(O)$ per class, until the quality reaches at least $q_{0}$.

Step 7: Return $S_{0}$. 


\section{Experimental Results}

The experiments were made using 7 databases from UCI [14] Repository of Machine Learning, with mixed and incomplete data. A description of these databases can be found in Table 1.

Table 1. Databases used in the experiments

\begin{tabular}{lcccc}
\hline \multicolumn{1}{c}{ Database } & Objects & Features & Missing Values & Classes \\
\hline Breast-cancer & 286 & 9 & 9 & 2 \\
Breast-cancer-breast & 286 & 9 & 9 & 2 \\
Credit-screening & 690 & 15 & 37 & 2 \\
Credit-screening-A9 & 690 & 15 & 37 & 2 \\
Credit-screening-A12 & 690 & 15 & 37 & 2 \\
Hepatitis & 155 & 19 & 75 & 2 \\
Import-85 & 205 & 25 & 46 & 7 \\
\hline
\end{tabular}

Breast-cancer-breast is the same database as Breast-cancer, but with the feature breast used as class feature. Credit-screening-A9 (Credit-screening-A12) is the same database as Credit-screening, but with the feature A9 (A12) used as class feature.

Each database was split taking $70 \%$ for training and $30 \%$ for testing. We repeated the process 5 times and average the results.

In our experiments we used a voting algorithm $A$ with the following parameters:

- The support sets system was the set of all typical testors of the training matrix.

- $\beta\left(\Omega O_{i}, \Omega O_{j}\right)=\frac{\sum_{\chi_{p} \in \Omega} C_{p}\left(\chi_{p}\left(O_{i}\right), \chi_{p}\left(O_{j}\right)\right)}{|\Omega|}$; partial similarity function, where $\Omega$ is a subset of features, $C_{p}$ the comparison criterion for the feature $\chi_{p}$, and $\chi_{p}\left(O_{i}\right)$ is the value of the feature $\chi_{p}$ in the object $O_{i}$.

- $C_{p}\left(\chi_{p}\left(O_{1}\right), \chi_{p}\left(O_{2}\right)\right)=\left\{\begin{array}{cc}1 & \text { if }\left|\chi_{p}\left(O_{1}\right)-\chi_{p}\left(O_{2}\right)\right|<\sigma_{p} ; \\ 0 & \text { otherwise }\end{array}\right.$ criteria for numeric features, where $\sigma_{p}$ is the standard deviation of the values of the feature $\chi_{p}$ in the training matrix.

- $C_{p}\left(\chi_{p}\left(O_{1}\right), \chi_{p}\left(O_{2}\right)\right)=\left\{\begin{array}{cc}1 & \text { if } \chi_{p}\left(O_{1}\right)=\chi_{p}\left(O_{2}\right) \\ 0 & \text { otherwise }\end{array} ;\right.$ similarity comparison criteria for non-numeric features.

- $\Gamma_{\Omega}\left(O_{p}, O\right)=\rho\left(O_{p}\right) \cdot \rho(\Omega) \cdot \beta\left(\Omega O_{p}, \Omega O\right)$; partial evaluation by rows for a fixed support set, where $\rho(\Omega)=\sum_{\chi_{i} \in \Omega} \rho\left(\chi_{i}\right)$. In the experiments $\rho\left(O_{p}\right)=1$ was used. 
- The weight of the feature $\chi_{i}$ is calculated, following [15], with the equation: $\rho\left(\chi_{i}\right)=\alpha \cdot P\left(\chi_{i}\right)+\beta \cdot L\left(\chi_{i}\right)$ with $\alpha, \beta>0$ and $\alpha+\beta=1$, being $\alpha$ and $\beta$ two parameters, which weight the influence of $P\left(\chi_{i}\right)$ and $L\left(\chi_{i}\right)$ respectively in $\rho\left(\chi_{i}\right)$. We used $\alpha=\beta=0.5 . P\left(\chi_{i}\right)$ is computed: $P\left(\chi_{i}\right)=\frac{\tau(i)}{\tau}$, where $\tau(i)$ is the number of typical testors, which contain the feature $\chi_{i}$ and $\tau$ the amount of all typical testors. $L\left(\chi_{i}\right)$ is computed: $L\left(\chi_{i}\right)=\frac{\sum_{t \in \Psi^{*}\left(\chi_{i}\right)} \frac{1}{|t|}}{\left|\Psi^{*}\left(\chi_{i}\right)\right|}$, where $\Psi^{*}\left(\chi_{i}\right)$ is the family of all typical testors, which contain the feature $\chi_{i}$.

- $\Gamma_{\Omega}^{j}(O)=\frac{1}{\left|K_{j}\right|} \sum_{O_{t} \in K_{j}} \Gamma_{\Omega}\left(O_{t}, O\right)$; partial evaluation by classes for a fixed support set.

- $\Gamma^{j}(O)=\frac{1}{\left|\Omega_{A}\right|} \sum_{\Omega \in \Omega_{A}} \Gamma_{\Omega}^{j}(O)$; total evaluation by classes.

- $\Gamma_{A}\left(\Gamma^{1}(O), \ldots, \Gamma^{r}(O)\right)=\left(\alpha_{1}^{A}(O), \ldots, \alpha_{r}^{A}(O)\right) ;$ decision $\quad$ - making rule, where $\alpha_{1}^{A}(O)=\left\{\begin{array}{cc}1 & \text { if } \Gamma^{i}(O) \geq \Gamma^{j}(O) \text { for } i \neq j \\ 0 & \text { otherwise }\end{array}\right.$.

- The quality function used, $Q_{A}\left(S_{0}, V\right)$, was the well classification ratio; hence, $W_{a}=1$ and $W_{o}=0$.

- The training matrix was used as validation matrix; $V=T$.

To make numerical comparisons, we denoted as GA the algorithm introduced in section 2 .

The accuracy of $A$ with the original matrix, CSE, ENN, GA, EALVOT and VOS are shown in Table 2 and 3. These results show that no method was clearly superior in all databases.

The compression ratios achieved by the algorithms are shown in Table 4.

It is clear that the proposed method reach higher compression ratios affecting lesser the classifier accuracy than any other methods.

Table 2. Accuracy of $A$ without editing compared with methods that do not use ALVOT

\begin{tabular}{lccc}
\hline \multicolumn{1}{c}{ Database } & $A$ & CSE & ENN \\
\hline Breast-cancer & 0.754 & 0.767 & 0.774 \\
Breast-cancer-breast & 0.58 & 0.624 & 0.547 \\
Credit-screening & 0.801 & 0.790 & 0.785 \\
Credit-screening-A9 & 0.739 & 0.744 & 0.734 \\
Credit-screening-A12 & 0.620 & 0.606 & 0.598 \\
Hepatitis & 0.738 & 0.691 & 0.740 \\
Import-85 & 0.495 & 0.498 & 0.451 \\
\hline
\end{tabular}


Table 3. Accuracy of $A$ without editing compared with methods that use ALVOT

\begin{tabular}{lccrc}
\hline \multicolumn{1}{c}{ Database } & $A$ & GA & EALVOT & \multicolumn{1}{c}{ VOS } \\
\hline Breast-cancer & 0.754 & 0.714 & 0.785 & 0.749 \\
Breast-cancer-breast & 0.58 & 0.522 & 0.560 & 0.578 \\
Credit-screening & 0.801 & 0.788 & 0.773 & 0.782 \\
Credit-screening-A9 & 0.739 & 0.738 & 0.717 & 0.705 \\
Credit-screening-A12 & 0.620 & 0.616 & 0.614 & 0.616 \\
Hepatitis & 0.738 & 0.738 & 0.740 & 0.743 \\
Import-85 & 0.495 & 0.422 & 0.460 & 0.489 \\
\hline
\end{tabular}

Table 4. Compression ratio

\begin{tabular}{lccccc}
\hline \multicolumn{1}{c}{ Database } & CSE & ENN & GA & EALVOT & VOS \\
\hline Breast-cancer & 0.29 & 0.21 & 0.51 & 0.27 & 0.60 \\
Breast-cancer-breast & 0.22 & 0.21 & 0.53 & 0.32 & 0.55 \\
Credit-screening & 0.50 & 0.10 & 0.50 & 0.13 & 0.60 \\
Credit-screening-A9 & 0.44 & 0.11 & 0.50 & 0.14 & 0.52 \\
Credit-screening-A12 & 0.21 & 0.24 & 0.49 & 0.42 & 0.48 \\
Hepatitis & 0.54 & 0.12 & 0.51 & 0.19 & 0.69 \\
Import-85 & 0.44 & 0.13 & 0.50 & 0.61 & 0.87 \\
\hline
\end{tabular}

\section{Conclusions}

ALVOT is a model for supervised classification based on partial precedences. It is mainly used in problems where the objects are described in terms of qualitative and quantitative features simultaneously, and some features have missing values.

Classifying with ALVOT turns expensive while the training matrix increases. In this paper a new object selection method (VOS) for ALVOT is introduced. It is based on the voting power of the objects, a magnitude calculated according the way ALVOT works. Several experiments were carried out and the comparisons with other methods showed that the proposed method reaches higher compression ratios affecting lesser the classifier accuracy than any other method.

\section{References}

1. Bezdek, J.C., Kuncheva, L.I.: Nearest Prototype classifiers designs: an experimental study. International Journal of Intelligent Systems 16 (2001) 1445 - 1473

2. Martínez Trinidad, J.F., Guzmán-Arenas, A.: The logical combinatorial approach to Pattern Recognition, an overview through selected works. Pattern Recognition 34 (2001) 741-751

3. Zhuravlev, Y.I., Nikiforov, V.V.: Recognition algorithms based on voting calculation. Journal Kibernetika 3 (1971) 1-11 
4. Ruiz-Shulcloper, J., Abidi, M.A.: Logical Combinatorial Pattern Recognition: A Review. In: Pandalai, S.G. (ed.): Recent Research Developments in Pattern Recognition. Transword Research Networks, USA (2002) 133-176

5. Gómez-Herrera, J., Rodríguez-Morán, O., Valladares-Amaro, S., Ruiz-Shulcloper, J., Pico-Peña, R.: Prognostic of Gas-oil deposits in the Cuban ophiological association, applying mathematical modeling. Geofísica Internacional 33 (1995) 447-467

6. Ortiz-Posadas, M.R.: Prognosis and evaluation of cleft palate patients' rehabilitation using pattern recognition techniques. World Congress on Medical Physics and Biomedical Engineering 35 (1997) 500

7. Carrasco-Ochoa, J.A., Martínez-Trinidad, J.F.: Editing and Training for ALVOT, an Evolutionary Approach. Lecture Notes in Computer Science 2690 (2003) 452-456

8. Carrasco-Ochoa, J.A., Martínez-Trinidad, J.F.: Combining Evolutionary Techniques to Improve ALVOT Efficiency. WSEAS Transactions on Systems 2 (2003) 1073-1078

9. Kuncheva, L.I., Bezdek, J.C.: Nearest prototype classification: clustering, genetic algorithms or random search. IEEE Transactions on Systems, Man and Cybernetics. Part C 28 (1998) 160-164

10. Decaestecker, C.: NNP: A neural net classifier using prototype. International Conference on Neural Networks, San Francisco, California (1993) 822-824

11. König, A., Rashhofer, R.J., Glesner, M.: A novel method for the design of radial-basisfunction networks and its implication for knowledge extraction. International Conference on Neural Networks, Orlando, Florida (1994) 1804-1809

12. García-Borroto, M., Ruiz-Shulcloper, J.: Selecting Prototypes in Mixed Incomplete Data. Lecture Notes in Computer Science 3773 (2005) 450-459

13. Wilson, D.L.: Asymptotic properties of nearest neighbor rules using edited data. IEEE Transactions on Systems, Man and Cybernetics 2 (1972) 408-421

14. Merz, C.J., Murphy, P.M.: UCI Repository of Machine Learning Databases. University of California at Irvine, Department of Information and Computer Science, Irvine (1998)

15. Lazo-Cortés, M., Ruiz-Shulcloper, J.: Determining the feature informational weight for non-classical described objects and new algorithm to calculate fuzzy testors. Pattern Recognition Letters 16 (1995) 1259-1265 